\title{
The National Bureau of Standards
}

\author{
announces a three-week course in \\ Radio Propagation
}

\author{
July 16 to August 3, 1962
}

The National Bureau of Standards will present a three-week course in Radio Propagation from July 16August 3, 1962, at the Boulder Laboratories, Boulder, Colorado.

The course, sponsored by the NBS Central Radio Propagation Laboratory in association with the University of Colorado, is designed to give scientists and engineers from universities, industry, and government agencies access to the latest advances in radio propagation research and show how this knowledge can best be applied to the design and development of communication systems. It will consider communication via the entire range of useable radio frequencies in the atmosphere, space, underground, and underwater, and will extend into the modes of propagation which are being explored for the future.

The NBS Central Radio Propagation Laboratory in Boulder conducts continuous studies designed to develop new methods of communication and more efficient use of the methods which now exist. This Laboratory is the central agency of the government for obtaining, analyzing, and disseminating information on the propagation of radio waves at all frequencies along the surface of the earth, in the atmosphere, and in space. The course is being offered as part of the Laboratory's responsibility to help industry and government design the most efficient communication systems possible.

The Departments of Electrical Engineering and Astrogeophysics of the University of Colorado are concerned with both academic and research programs in electromagnetic theory and atmospheric physics. To facilitate transfer of academic credit for students working on graduate degrees in areas related to radio propagation, the course will be included in the Graduate program at the University of Colorado. Such students must plan an additional half day at the end of the course to write a final examination

The 1962 course will employ a unified approach to both ionospheric and tropospheric propagation. This unification permits adoption of the experience and suggestions obtained from the 1961 course. In particular, increased emphasis will be given to both fundamental physies and to systems applications. An integrated development of tropospheric and ionospheric propagation will stress similarities and differences of the two. Lectures will be supplemented by regularly scheduled informal discussions conducted by all lecturers within a particular topic group.

Prerequisites for the course are a bachelor's degree in Electrical Engineering, Physics, or other suitable academic or practical experience. The tuition is $\$ 300$ for the entire course running from July 16 through August 3; a small additional University registration fee will be required of students who also desire academic credit.

Registration will be limited and early application should be made to insure consideration. To facilitate local arrangements, registration will be closed July 1, 1962. Further details of the course and registration forms are available from: Edmund H. Brown, Education Director, Boulder Laboratories, National Bureau of Standards, Boulder, Colorado.

\section{Following is a list of tentative lectures:}

1. Historical Development of Maxwell's Equations

2. The Wave Solutions of Maxwell's Equations

3. Properties of the Atmosphere I

4. Properties of the Atmosphere II

5. Measurement of Atmospheric Properties

6. Ray Theory in Radio Propagation

7. Statistics in Radio Propagation

8. Random Processes in the Medium

9. Turbulent Scattering Theory

10. Source of Noise I

11. Source of Noise II
12. Experimental Methods I
13. Experimental Methods II

14. Line-of-Sight Tropospheric Propagation

15. Tropospheric Refraction and Attenuation

16. Diffraction and Obstacle-Gain

17. Irregular Terrain

18. Tropospheric Turbulence

19. Line-of-Sight Phase Stability

20. Tropospheric Forward Scatter I

21. Tropospheric Forward Scatter II

22. Formation and Structure of the Ionosphere

23. The Quiet Ionosphere

23. The Quiet Ionosphere

24. The Disturbed Ionosphere

25. Geomagnetism and Geom
26. Magneto-Ionic Theory

26. Magneto-Ionic Theory
27. Applications of the Appleton-Hartree Formula

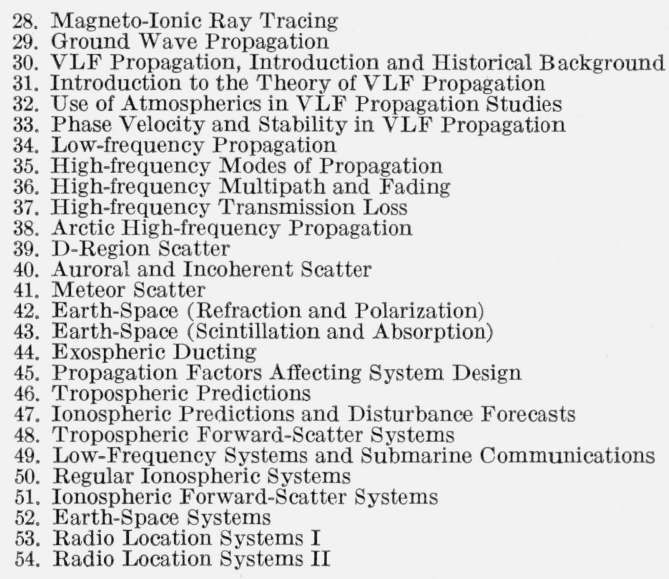

